\title{
Advantages of Enzyme Could Lead to Improved Biofuels Production
}

Highlights in

Science

Cellulase C. bescii CelA, a highly active and stable enzyme, exhibits a new cellulose digestion paradigm promoting inter-cellulase synergy.
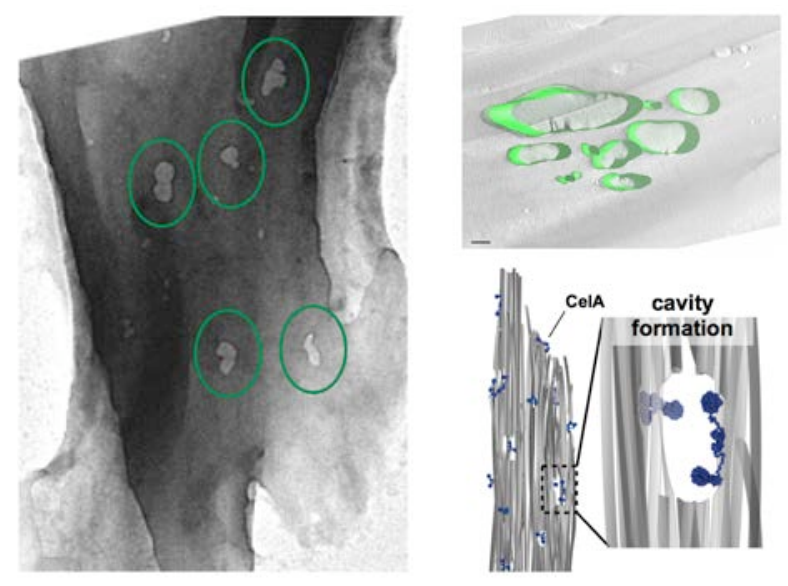

Transmission electron micrographs and schematic of partially digested small Avicel particles. Particles digested to approximately $65 \%$ conversion with CelA display surface cavities of various sizes. All scale bars are $500 \mathrm{~nm}$. Image by Bryon Donohoe, NREL

\section{University of Georgia, isolated}

the thermophilic cellulase CelA from C. bescii. A comparison was conducted of its cellulolytic activity with that of a binary mixture containing both T. reesei Cel7A exoglucanase and $A$. cellulolyticus Cel5A endoglucanase on several substrates. The researchers also compared the cellulose digestion mechanisms of these two enzyme systems using electron microscopy and modeling.

CelA was shown to retain high activity at all temperatures tested, converting $60 \%$ of glucan at $85^{\circ} \mathrm{C}$ compared to $28 \%$ glucan conversion for the common exo/endo cellulase standard mixture, Cel7A/Cel5A, at its optimal temperature of $50^{\circ} \mathrm{C}$. This difference in activity translates to a seven-fold increase in activity for CelA at the molecular level.

Transmission electron microscopy studies of cellulose following incubation with CelA suggest that CelA is capable of not only the common surface ablative mechanism driven by general cellulase processivity, but also of excavating extensive cavities into the surface of the substrate. Additionally, during the digestion experiments, CelA achieved $60 \%$ conversion of xylan in native switchgrass, showing its potential for industrial processes using mild or no pretreatment.

Technical Contact: Yannick Bomble,yannick.bomble@nrel.gov

Reference: Brunecky, R.; Alahuhta, P.; Xu, Q.; Donohoe, B.; Crowley, M.; Kataeva, I, Yang, SJ, Adams, M; Lunin, V.; Himmel, M.; Bomble, Y. (2013). "Revealing Nature's Cellulase Diversity: The Digestion Mechanism of Caldicellulosiruptor bescii CelA." Science (342:6165); pp. 1513-1516. DOI: 10.1126/science.1244273.

\section{Key Research Results}

\section{Achievement}

The research team isolated CelA, a highly active cellulase with a novel cellulose digestion mechanism. The X-ray structures of the primary protein components of CelA were also determined, advancing the understanding of the mode of action of this cellulase.

\section{Key Result}

CelA was shown to retain high activity at all temperatures tested, converting $60 \%$ of glucan compared to $28 \%$ glucan conversion for the more common exo/endo cellulase standard mixture, Cel7A/Cel5A, at its optimal temperature of $50^{\circ} \mathrm{C}$.

\section{Potential Impact}

CelA and similar multi-functional cellulases represent a new and distinct paradigm for cellulose digestion. This mechanism is fundamentally different from conventional cellulases and could help increase inter-cellulase synergy in consolidated bioprocessing microorganisms, as well as in commercial cellulase formulations used for biofuels production.
NREL is a national laboratory of the U.S. Department of Energy, Office of Energy Efficiency and Renewable Energy, operated by the Alliance for Sustainable Energy, LLC.

15013 Denver West Parkway Golden, CO 80401 303-275-3000 | www.nrel.gov

NREL/FS-2700-61022 | January 2014 